\title{
Lesiones Orales en Tejidos Blandos de una Población Escolar en la Ciudad de México
}

\author{
Orales Lesions in Soft Tissues of a School Population in Mexico City
}

Tomás Caudillo Joya'; María del Pilar Adriano Anaya1 \& Pilar Alejandra Caudillo Adriano²

CAUDILLO, J. C.; ADRIANO, A. M. P. \& CAUDILLO, A. P. A. Lesiones orales en tejidos blandos de una población escolar en la Ciudad de México. Int. J. Odontostomat., 12(2):177-181, 2018.

RESUMEN: Identificar la distribución y frecuencia de las lesiones orales en los tejidos blandos de la población escolar de seis a doce años de diez escuelas primarias de la delegación Iztapalapa en la Ciudad de México, es el propósito de este estudio de tipo descriptivo, transversal y observacional realizado en una muestra por conveniencia de 2394 escolares de seis a doce años, que entregaron su permiso firmado previa información a los padres de familia. La información se capturó en el Programa de Cómputo SPSS versión 17.0. El 85,6 \% de los escolares participantes se encontraron libres de lesiones orales en sus tejidos blandos. Las lesiones traumáticas, fueron las de mayor frecuencia ya que 183 de ellos (7,75), las presentaron, ya sea en carrillo, labio o en la lengua. El contar con estudios que nos permitan identificar las lesiones en los tejidos blandos en la población escolar es de suma importancia debido a que se podrá actuar con programas específicos para su control y prevención. Con respecto a la prueba $t=0,001$, se encontró significancia estadística entre el sexo y la distribución y localización de las lesiones orales en los tejidos blandos de los escolares.

PALABRAS CLAVE: tejidos blandos, mucosa, Escolares, epidemiología.

\section{INTRODUCCIÓN}

Hasta la actualidad en el mundo se han realizado pocos estudios epidemiológicos sobre la Patología de la mucosa oral en niños y adolescentes (Redman, 1970), así mismo en México esto se refleja ya que no existen suficientes estudios que orienten hacia la epidemiología de las lesiones de la mucosa oral en pacientes pediátricos (Espinoza-Zapata et al., 2006). Los estudios que analizan la prevalencia de las enfermedades orales están en su mayoría asociados hacia la edad adulta y por tanto en la literatura mundial existen relativamente pocos reportes que comenten acerca de las condiciones de la mucosa oral y de las enfermedades pediátricas (Majorana et al., 2010). aunque el examen de cavidad oral en niños proporciona importantes herramientas en el diagnóstico de las alteraciones del desarrollo, enfermedades neoplásicas, infecciosas e inflamatorias (González González et al., 2011).

Rioboo-Crespo et al. (2005) comentaron que los porcentajes de las lesiones en tejidos blandos en la población infantil varían notablemente, encontrando cifras que van desde el 4,1 \% hasta el 52,6 \%, siendo la estomatitis aftosa recurrente, herpes labial, lengua geográfica, candidiasis y lesiones de origen traumático las más prevalentes, estas diferencias metodológicas se evitarían aplicando protocolos internacionales que uniformizaran los criterios a seguir.

Arendorf \& van der Ross (1996) realizaron un estudio en Sudáfrica, encontrando la incidencia de Úlcera traumática en un 2,5\%; mientras que otro estudio en Israel, (Ulmansky et al., 1999), señalan incidencia de Linfoma de Burkitt en un 60,0 \% y Papilomas y Fibromas en $72,5 \%$.

En un estudio realizado en México (Reynoso Licona \& Mendoza Núñez, 2004), la tasa de prevalencia de lesiones en tejidos blandos en niños de 1 a 6 años de edad fue alta 44,0\% (466), Se encontró una frecuencia mayor en el género masculino $48,67 \%$, que en el femenino $38,32 \%$. La glositis

${ }^{1}$ Facultad de Estudios Superiores Zaragoza Universidad Nacional Autónoma de México, México.

${ }^{2}$ Universidad Autónoma Metropolitana Xochimilco, México. 
migratoria benigna se encontró con una prevalencia de $5,49 \%$. Las lesiones herpéticas su prevalencia fue del $5,96 \%$. La úlcera traumática se identificó como la forma de mayor frecuencia debido a la enorme cantidad de factores locales, se registró en un $12,39 \%$, Esta lesión se localizó simultáneamente en encía superior e inferior y fue la de mayor prevalencia con un $10,03 \%$.

En la Universidad Central de Venezuela (Jiménez et al., 2008), se realizó un estudio retrospectivo en niños y adolescentes de 1992-2006, determinando lesiones más frecuentes en el grupo etáreo, género y localización en cavidad oral resultando un total de 944 pacientes en un período de 15 años, la lesión más frecuente fue el Mucocele $(19,60$ $\%)$, seguida por Fibroma Traumático (10,28 \%), Virus Herpes tipo I (8,26\%), Granuloma Piogénico $(7,10$ $\%)$ y Papiloma oral $(6,89 \%)$; el género femenino predomina con (54,66\%), con respecto al masculino de $(45,34 \%)$. El grupo etario más afectado fue entre los 13 a 16 años (32\%), siendo las localizaciones más frecuentes: labio inferior (35\%), encía (27\%), carriIlo (12\%), labio superior (10\%), lengua y paladar (8 $\%)$.

En Uruguay se identificó la frecuencia de enfermedades en la mucosa oral en 401 individuos de 0 a 14 años, distribuidos en dos muestras aleatorias, provenientes del Instituto Nacional del Niño y Adolescente del Uruguay (INAU) y el Centro de Asistencia del Sindicato Médico del Uruguay (CASMU) (Keochgerián et al., 2009). La prevalencia total fue de $32,9 \%$; en CASMU 36,8 \% y en INAU $29 \%$. La prevalencia de lesiones traumáticas en el INAU fue del $4,5 \%$ y en CASMU de $18,4 \%$.

Es importante evaluar las lesiones de la mucosa oral en este grupo de población ya que determinadas enfermedades sistémicas son provocadas por diferentes factores entre ellos orales (Sedano, 1975; Ruiz et al., 2009), aumentando el riesgo de enfermedades crónicas como las enfermedades cardiovasculares y cerebrovasculares, la diabetes mellitus y enfermedades respiratorias (Mejía González et al.,2012).

Sin embargo no existen métodos estandarizados para su diagnóstico, aunque la Organización Mundial de la Salud (OMS) ha incrementado sus recomendaciones epidemiológicas en este grupo de población estableciendo signos y síntomas de la mucosa oral propias de los infantes que pueden cambiar con la edad y ser diferentes a la de los adultos (Kramer et al., 1980; Organización Mundial de la Salud., 1995). Es por eso la importancia de realizar este estudio en la población escolar de seis a doce años de diez escuelas primarias públicas de la delegación Iztapalapa localizada al oriente de la Ciudad de México, en zonas de alta marginación, con el propósito de identificar las lesiones orales en tejidos blandos y su localización con respecto a los criterios de la OMS.

\section{MATERIAL Y MÉTODO}

El estudio fue transversal, observacional y descriptivo conformado por una muestra por conveniencia de 2394 escolares que entregaron su permiso firmado previa información a los padres de familia de los objetivos del proyecto, para participar en este estudio. Las diez primarias en las que se llevó a cabo el estudio se encuentran dentro del programa de salud oral que la Facultad de Estudios Superiores Zaragoza UNAM está desarrollando en la delegación Iztapalapa, ubicada en la zona oriente de la Ciudad de México.

En cada uno de los escolares que conformaron la muestra, se llevó a cabo el examen de la mucosa oral y de los tejidos blandos siguiendo el orden y las recomendaciones de la OMS (1997), por cinco pasantes y dos Maestros previamente entrenados y estandarizados en el manejo y detección de las lesiones en los tejidos blandos de los escolares, registrando en una ficha epidemiológica la edad, sexo, el trastorno oral y su localización (Kramer et al.). Las barreras de protección fueron las planteadas por la Norma Oficial Mexicana para el control y manejo de infecciones orales (Norma Oficial Mexicana NOM087-ECOL-SSA1-2002, 2002). La información se capturó posteriormente en el Programa de Cómputo SPSS versión 17.0

\section{RESULTADOS}

El número de escolares que participaron en este estudio fue de 2394, entre las edades de seis a doce años, de los cuales el $51 \%$ (1218) fueron del sexo masculino y el $49 \%$ (1176), del femenino (Tabla I).

El $83,2 \%$ de los escolares se encontraron libres de lesiones en sus tejidos blandos. Del $100 \%$ de 
Tabla I. Distribución de los escolares por edad y sexo.

\begin{tabular}{ccc}
\hline Edad & No. & $\%$ \\
\hline 6 & 235 & 9.8 \\
7 & 376 & 15.7 \\
8 & 369 & 15.4 \\
9 & 413 & 17.3 \\
10 & 408 & 17.0 \\
11 & 379 & 15.8 \\
12 & 214 & 8.9 \\
Masculino & 1218 & 51 \\
Femenino & 1176 & 49 \\
Total & 2394 & 100.0 \\
\hline
\end{tabular}

los escolares, $365(17,75 \%)$, presentaron algún tipo de lesión en los tejidos blandos de la boca, de los cuales $214(10,41 \%)$ corresponde al sexo masculino y $151(7,34 \%)$ al femenino; $187(9,2 \%)$, escolares presentaron lesiones traumáticas en carrillos, labios o lengua, siendo esta la de mayor frecuencia.

Con respecto a la prueba $t=0.001$, se encontró significancia estadística entre el sexo y la distribución y localización de las lesiones orales en los tejidos blandos de los escolares (Tabla II).

La edad en que se presentaron más lesiones fue la de nueve años con 80 escolares afectados, seguida la de diez con 65 escolares con algún tipo de lesión. Encontrando significancia entre estas dos variables $\mathrm{X}^{2}=0.020$ (Tabla III).

\section{DISCUSION}

El identificar las lesiones en los tejidos blandos orales más frecuentes en población escolar de seis a doce años, es de suma importancia para tener diagnósticos sobre esta problemática y conocer cuáles son los problemas que aquejan a esta población ya que la mayoría de los estudios han sido realizados en población adulta y no debemos inferir que se tratan de las mismas lesiones.

En México los problemas orales que más se han estudiado es la caries dental, periodontopatías y las maloclusiones en la población escolar, siendo muy pocos los estudios de las lesiones en tejidos blandos en la edad escolar, en consecuencia no contamos con un perfil epidemiológico de éste problema.

La epidemiología de las enfermedades de la cavidad oral ha estado ubicada en un plano secundario, sin recibir la atención apropiada en la planificación del servicio de salud oral (Pindborg, 1977; Jiménez Palacios et al., 2009). Por tal motivo existen pocas referencias sobre la incidencia y tipos de lesiones en los tejidos blandos que se presentan en niños y adolescentes.

La prevalencia de lesiones en tejidos blandos orales en este estudio fue del $17,75 \%$ en los escolares. De los 365 (100\%), escolares que presentaron alguna lesión, 187 (51\%), presentó lesiones traumáticas ya sea en carrillos, labios o lengua, que puede ser provocado por hábitos o estrés; 75 (20,5

Tabla II. Trastornos y localización de lesiones orales de los tejidos blandos de los escolares por sexo.

\begin{tabular}{lcccccc}
\hline Lesión & No, & $\%$ & $\mathrm{M}$ & $\%$ & $\mathrm{~F}$ & $\%$ \\
\hline lesiones traumáticas mordida en carrillo & 160 & 7,9 & 88 & 4,3 & 72 & 3,6 \\
lesiones traumáticas mordida labio & 19 & 0,9 & 12 & 0,6 & 7 & 0,3 \\
lesiones traumáti cas en lengua & 8 & 0,4 & 4 & 0,2 & 4 & 0,2 \\
úlcera aftosa en labio & 65 & 3,2 & 38 & 1,9 & 27 & 1,3 \\
fístula en suelo de boca & 20 & 1 & 17 & 0,8 & 3 & 0,2 \\
frenillo lengua & 11 & 0,5 & 10 & 0,46 & 1 & 0,04 \\
absceso en fondo de saco inferior & 41 & 2 & 24 & 1,2 & 17 & 0,8 \\
absceso en fondo de saco superior & 14 & 0,7 & 9 & 0,4 & 5 & 0,3 \\
glositis migratoria benigna (lengua geográfica) & 9 & 0,4 & 2 & 0,1 & 7 & 0,3 \\
lengua pigmentada & 5 & 0,2 & 2 & 0,1 & 3 & 0,1 \\
pigmentación en encía & 5 & 0,2 & 2 & 0,1 & 3 & 0,1 \\
úlcera por aparato de ortodoncia en carrillos & 2 & 0,1 & 0 & 0 & 2 & 0,1 \\
resequedad en labios & 5 & 0,2 & 5 & 0,2 & 0 & 0 \\
verruga en labio & 1 & 0,05 & 1 & 0,05 & 0 & 0 \\
Total & 365 & 17,75 & 214 & 10,41 & 151 & 7,34 \\
& & & & & $t$ & $t$ \\
\end{tabular}


Tabla III. Distribución de lesiones en tejidos blandos por edad de los escolares.

\begin{tabular}{|c|c|c|c|c|c|c|c|c|}
\hline Lesiones & & & & Edac & & & & \\
\hline & 6 & 7 & 8 & 9 & 10 & 11 & 12 & Total \\
\hline lesiones traumáticas mordida labio & 2 & 2 & 1 & 2 & 8 & 1 & 3 & 19 \\
\hline lesiones traumáticas mordida en carrillo & 20 & 25 & 17 & 31 & 29 & 23 & 15 & 160 \\
\hline lesiones traumáti cas en lengua & 1 & 4 & 1 & 2 & 0 & 0 & 0 & 8 \\
\hline absceso en fondo de saco inferior & 4 & 8 & 4 & 11 & 3 & 4 & 7 & 41 \\
\hline absceso en fondo de saco superior & 0 & 1 & 1 & 3 & 4 & 3 & 2 & 14 \\
\hline fístula en suelo de boca & 3 & 1 & 4 & 2 & 3 & 5 & 2 & 20 \\
\hline lengua geográfica o glositis migratoria benigna & 0 & 0 & 0 & 6 & 2 & 1 & 0 & 9 \\
\hline lengua pigmentada & 1 & 1 & 0 & 3 & 0 & 0 & 0 & 5 \\
\hline úlcera aftosa en labio & 1 & 7 & 5 & 17 & 11 & 13 & 11 & 65 \\
\hline pigmentación en encía & 0 & 1 & 0 & 2 & 2 & 0 & 0 & 5 \\
\hline frenillo lengua & 0 & 1 & 2 & 1 & 1 & 4 & 2 & 11 \\
\hline resequedad en labios & 0 & 0 & 0 & 0 & 2 & 1 & 2 & 5 \\
\hline verruga en labio & 0 & 0 & 0 & 0 & 0 & 1 & 0 & 1 \\
\hline úlcera en carrillo por aparato de ortodoncia & 0 & 0 & 0 & 0 & 0 & 1 & 1 & 2 \\
\hline Subtotal & 32 & 51 & 35 & 80 & 65 & 57 & 45 & 365 \\
\hline Total & 20 & 325 & 334 & 332 & 342 & 322 & 169 & 2027 \\
\hline
\end{tabular}

$\%)$, escolares tuvieron abscesos o fístulas consecuencia de lesiones cariosas no tratadas que se convirtieron en crónicas provocando esta manifestación y si no se resuelven pueden llegar a provocar algún problema sistémico como artritis reumatoide y cardiopatías.

Las lesiones encontradas podríamos considerarlas como benignas en esta etapa de la vida debido a que no ponen en riesgo la vida de los escolares.

Del total de escolares revisados (2394), Con respecto al sexo el más afectado $(10,41 \%)$ fue el masculino mientras que el $(7,34 \%)$ corresponde al femenino, estos resultados nos invita a seguir estudiando este problema para profundizar en su distribución y frecuencia por sexo.

Con respecto a la edad las más afectadas fueron los escolares de 9 y 10 años, probablemente a que son más inquietos.

Los resultados de éste estudio fueron diferentes al realizado por Shulman (2005) quién encontró que la lesión más prevalente fue el mordisqueo de mejilla, mientras que en el estudio realizado en la Universidad Central de Venezuela la lesión más frecuente fue el Mucocele (19,60 \%) (Keochgerián et al.). En Uruguay, la prevalencia de lesiones traumáticas en el INAU fue del $4,5 \%$ y en CASMU de $18,4 \%$ (Ruiz et al.). En los dos últimos estudios la prevalencia de estas lesiones fueron más elevadas que en el estudio realizado en la delegación Iztapalapa, en la Ciudad de México.

\section{CONCLUSIONES}

Las principales lesiones en este estudio fueron las traumáticas y los abscesos. Su presencia fue más prevalente en el sexo masculino.

Las lesiones en los tejidos blandos orales en la población infantil, son diferentes a la población adulta, por lo que se deben plantear criterios universales para su estudio en la población infantil, e identificar su distribución y frecuencia y como odontólogos poder actuar con programas específicos preventivos y curativos para el control de los mismos. Así mismo es importante seguir investigando en este grupo de población ya que su estudio en México ha sido limitado.

CAUDILLO, J. C.; ADRIANO, A. M. P. \& CAUDILLO, A. P. A. Oral lesions in soft tissues of a school population in Mexico City. Int. J. Odontostomat., 12(2):177-181, 2018.

ABSTRACT: The aim of the study was to identify the distribution and frequency of oral injuries in soft tissues in a school population of six to twelve year-old children, in ten elementary schools of the Iztapalapa delegation in Mexico city. The study type was descriptive, transversal and observational and was carried out in a sample of 2394 
schoolchildren from six to twelve years of age, with prior consent signature from the parents or guardians. The information was captured in the software SPSS vesion 17.0. Of the schoolchildren participating in the study, $85.6 \%$ were found to be free of soft tissue injuries. Traumatic injuries were the most frequent as 183 of them (7.75), presented in the cheek, lip or tongue. Studies that identify lesions in soft tissues in school children are important because they are beneficial in establishing specific control and prevention programs in these populations. Statistical significant differences were found between sexes, distribution and site of oral injuries in soft tissues of the population studied.

KEY WORDS: soft tissues; mucosa; school children; epidemiology.

\section{REFERENCIAS BIBLIOGRÁFICAS}

Arendorf, T. M. \& van der Ross, R. Oral soft tissue lesions in a black pre-school South African population. Community Dent. Oral Epidemiol., 24(4):296-7, 1996.

Espinoza-Zapata, M.; Loza-Hernández, G. \& Mondragón-Ballesteros, R. Prevalencia de lesiones de la mucosa bucal en pacientes pediátricos. Informe preliminar. Rev. Cir. Cir., 74(3):1537, 2006.

González González, R.; Bologna Molina, R.; Nevárez Rascón, A. \& Carreón Burciaga, R. G. Lesiones frecuentes de la mucosa bucal en niños y adolescentes: Revisión literaria. Rev. ADM., 68(1):17-24, 2011.

Jiménez Palacios, C.; Ramírez, R.; Ortiz, V.; Virgüez, Y. \& Benítez, A. Identificación de lesiones en los tejidos blandos de la cavidad bucal en adolescentes con privación de libertad en el odontopediátrico de carapa. Septiembre 2005-abril 2006 Venezuela. Acta Odontol. Venez., 47(2):327-33, 2009.

Jiménez, C.; Kilikan, R.; Pérez, C.; Herrera, N. \& Hernández, L. Estudios retrospectivo de lesiones patológicas en niños y adolescentes. Rev. Latinoam. Ortod. Odontopediatr. [Revista en Internet] 2008. Disponible en: https://www.ortodoncia.ws/publicaciones/2008/art-6/

Keochgerián, V.; Cuestas, M.; Padula, D. \& Scavino, M. Frecuencia de las enfermedades de la mucosa bucal en el Uruguay en niños de 0 a 14 años. Odontoestomatología, 11(13):43-57, 2009.

Kramer, I. R.; Pindborg, J. J.; Bezroukov, V. \& Infirri, J. S. Guide to epidemiology and diagnosis of oral mucosal diseases and conditions. World Health Organization. Community Dent. Oral Epidemiol., 8(1):1-26, 1980.

Majorana, A.; Bardellini, E.; Flocchini, P.; Amadori, F.; Conti G. \& Campus, G. Oral mucosal lesions in children from 0 to 12 years old: ten years' experience. Oral Surg. Oral Med. Oral Pathol. Oral Radiol. Endod., 110(1):e13-8, 2010.

Mejía González, A. M.; Lomelí Buyoli, G. \& Gaxiola Cortés, M. C. Manual de Procedimientos Estandarizados para la Vigilancia Epidemiológica de las Patologías Bucales. Ciudad de México, Secretaría de Salud, Subsecretaría de Prevención y Promoción de la Salud, Dirección General de Epidemiología, 2012. pp.13-4.

NORMA Oficial Mexicana NOM-087-ECOL-SSA1-2002, Protección ambiental - Salud ambiental - Residuos peligrosos biológico-infecciosos - Clasificación y especificaciones de manejo. Ciudad de México, Secretaría de Salud, Gobierno de México,
2002. Disponible en: http://www.salud.gob.mx/unidades/cdi/ nom/087ecolssa.html.

Organización Mundial de la Salud (OMS). Encuestas Básicas de Salud Bucodental. Métodos Básicos. $4^{\mathrm{a}}$ ed. Ginebra, Organización Mundial de la Salud, 1997. pp.1-27.

Pindborg, J. J. Epidemiology and public health aspects of diseases of the oral mucosa. J. Dent. Res., 56 Spec. No.:C14-9, 1977.

Redman, R. S. Prevalence of geographic tongue, fissured tongue, median rhomboid glossitis, and hairy tongue among 3,611 Minnesota schoolchildren. Oral Surg. Oral Med. Oral Pathol., 30(3):390-5, 1970.

Reynoso Licona, A. \& Mendoza Núñez, V. M. Magnitud de lesiones bucales de tejidos blandos en niños de 1 a 6 años de edad de la ciudad de México. Rev. ADM, 61(2):65-9, 2004.

Rioboo-Crespo, M. del R.; Planells-del Pozo, P. \& Rioboo-García, $\mathrm{R}$. Epidemiology of the most common oral mucosal diseases in children. Med. Oral Patol. Oral Cir. Bucal, 10(5):376-87, 2005.

Ruiz, O.; Estupinan, S.; Miller, T.; Soto, A. \& Hernández, F. Módulo Salud Oral. Salud del Niño y del Adolescente. Salud Familiar y Comunitaria. Organización Panamericana de la Salud, Atención Integrada a las Enfermedades Prevalentes de la Infancia, 2009. Disponible en: http://new.paho.org/hq/ dmdocuments/2009/si-oral1.pdf

Sedano, H. O. Congenital oral anomalies in argentinian children. Community Dent. Oral Epidemiol., 3(2):61-3, 1975.

Shulman, J. D. Prevalence of oral mucosal lesions in children and youths in the USA. Int. J. Paediatr. Dent., 15(2):89-97, 2005.

Ulmansky, M.; Lustmann, J. \& Balkin, N. Tumors and tumor-like lesions of the oral cavity and related structures in Israeli children. Int. J. Oral Maxillofac. Surg., 28(4):291-4, 1999.

Dirección para correspondencia:

María del Pilar Adriano Anaya

Facultad de Estudios Superiores Zaragoza

Universidad Nacional Autónoma de México

MÉXICO

Email: adriano124@hotmail.com

Recibido : 23-11-2017

Aceptado: 02-05-2018 